\title{
Democracia y derecho a decidir Democracy and the right to decide
}

\author{
Josep M. Vilajosana \\ Universitat Pompeu Fabra \\ ORCID ID 0000-0001-8416-2418 \\ josep.vilajosana@upf.edu
}

\section{Cita recomendada:}

Vilajosana, J. M. (2020). Democracia y derecho a decidir. Eunomía. Revista en Cultura de la Legalidad, 18, pp. 375-391.

doi: https://doi.org/10.20318/eunomia.2020.5284

Recibido / received: 24/02/2020

\begin{abstract}
Resumen
En este artículo, el autor defiende el derecho a decidir, concretado así: un referéndum sobre el futuro de Cataluña no está prohibido en la Constitución española. Esta tesis se sustenta en que tanto el principio de indisolubilidad (art. 2.1. CE) como el de la soberanía nacional (art. 1.2. CE) deben ponderarse adecuadamente con los principios definitorios de una democracia liberal (arts. 1.1., 23.1, 9 y $10 \mathrm{CE}$ ). Desde esta perspectiva, el trabajo ofrece razones para justificar dos cuestiones importantes: 1) la posibilidad de realizar un referéndum sobre la independencia de Cataluña, teniendo en cuenta una interpretación evolutiva de los derechos democráticos vinculados a una concepción densa de la democracia; 2) la posibilidad de que esa consulta tenga como sujetos a los catalanes, con el fin de evitar pasar del principio de la mayoría al dominio de la mayoría.
\end{abstract}

\section{Palabras clave}

Legalidad, legitimidad, principios, democracia, derecho a decidir, Constitución.

\begin{abstract}
In this article, the author defends the right to decide, meaning that holding a referendum on the political future of Catalonia is not banned by the Spanish Constitution (SC). The principal reason cited is that the principle of indissolubility (section 2.1 of the SC) and the principle of national sovereignty (section 1.2 SC) should be adequately balanced with the principles of liberal democracy (as defined in sections 1.1., 23.1., 9 and 10 of the SC). In light of this perspective, the article provides justification for two main aspects: 1) holding a referendum on Catalonia's independence on the grounds of an evolutive interpretation of democratic rights linked to a dense conception of democracy; and 2) holding a referendum exclusively in Catalonia, in order to avoid shifting from the principle of the majority to the dominion of the majorities.
\end{abstract}

\section{Keywords}

Legality, legitimacy, principles, democracy, right to decide, Constitution. 
SUMARIO. 1. Introducción. 2. Legalidad y legitimidad en el Estado constitucional contemporáneo. 3. Derechos humanos y principios constitucionales. 4. Derecho a decidir como derecho de la ciudadanía a ser consultada sobre su futuro político. 5. La ponderación de los principios relevantes. 5.1. El principio democrático como límite al principio de indisolubilidad. 5.1.1. Una interpretación evolutiva de los derechos democráticos. 5.1.2. Una concepción densa de la democracia. 5.2. El principio democrático como límite al principio de la soberanía nacional. 5.2.1. La determinación del demos. 5.2.2. Principio de la mayoría y dominio de la mayoría. 5.2.3. Los principios de autonomía, inviolabilidad y dignidad de la persona. 5.2.4. Alcance ponderado de la soberanía nacional.

\section{Introducción}

Las recientes iniciativas promovidas por el primer gobierno de coalición del Estado español y por un sector de los representantes soberanistas tal vez contribuyan a generar un clima en el que se pueda discutir desapasionadamente sobre las distintas cuestiones que han emergido del llamado proceso político catalán, algo que por ahora no se ha producido ni en el ámbito político ni en el académico. No soy muy optimista al respecto (ni en lo político ni en lo académico), pero me hubiera parecido una descortesía rechazar la amable invitación a participar en el debate académico que he recibido por parte del director de Eunomía, José María Sauca. Le agradezco, pues, que haya contado conmigo en esta loable iniciativa y solo espero que mi contribución no le defraude enteramente.

Las transformaciones que está produciendo el mencionado proceso forman un banco de pruebas inigualable para diversas materias de estudio, como por ejemplo el Derecho constitucional, la teoría del Derecho y la filosofía jurídico-política. Esos cambios se enmarcan en una pregunta central que hay que responder, por supuesto desde la esfera política, pero también desde el ámbito académico. Puede formularse de este modo: ¿qué respuesta debe dar un Estado democrático de Derecho en el siglo XXI ante una petición continuada y pacífica de una parte muy relevante de la población de una comunidad interna que reivindica el derecho a decidir sobre su futuro político, incluyendo la posibilidad de independizarse? Hasta ahora, los distintos poderes del Estado español han dado una contestación a este interrogante muy distinta de la que han ofrecido otros países democráticos como Reino Unido o Canadá. En efecto, en vez de autorizar, delegar o simplemente tolerar un referéndum, el Estado español ha optado por la negativa a pactar una consulta y, cuando esta efectivamente se ha producido, aplicar una legislación de excepción a través del art. 155 CE y tratar esas reivindicaciones políticas por la vía penal. Este camino ha conducido, por el momento, a condenas de algunos líderes independentistas con penas por delitos de sedición y malversación, que algunos juristas consideran injustificadas. El resultado de ello ha sido entrar de lleno en una crisis institucional de consecuencias inciertas. Pero acotemos algo más el alcance del proceso político que está en la base de aquel interrogante.

Como inicio de esa senda puede tomarse la tramitación de la Reforma del Estatuto de autonomía que acabó siendo aprobada por el Parlament en 2006, o, si se prefiere, la publicación de la sentencia 31/2010 del Tribunal Constitucional sobre el 
Estatut. A partir de este momento se ha ido consolidando claramente un movimiento social que ha puesto en el primer punto de la agenda política la aspiración y la posibilidad de la independencia de Cataluña, sobre todo a partir de la reclamación ampliamente compartida por la sociedad catalana del llamado "derecho a decidir". Bastará con recordar las manifestaciones multitudinarias del 10 de julio de 2010 (con el lema "Somos una nación. Nosotros decidimos"), la del 11 de septiembre de 2012 (con el lema "Cataluña, nuevo estado de Europa"), la de la vía catalana hacia la independencia de la Diada de 2013 y, desde entonces, todas las que se han ido produciendo cada 11 de septiembre hasta la fecha.

Es preciso subrayar el sentido que ha tenido este proceso, frente a quienes sostienen que el movimiento originado en Cataluña ha supuesto desde su inicio un ataque a la soberanía nacional. Actualmente parece que efectivamente la cuestión de fondo tiene que ver con la soberanía. Ahora bien, no fue así cuando se inició la reforma del Estatuto catalán hace quince años. En aquel momento se siguieron escrupulosamente los pasos procedimentales previstos en la CE para tal reforma y esta se aprobó en 2006. Luego vendría la presentación de sendos recursos de inconstitucionalidad promovidos por el Partido Popular y por el Defensor del Pueblo (acompañada de una campaña que precisamente intentaba hacer pasar lo que era un procedimiento de reforma dentro del marco autonómico como un atentado a la soberanía nacional) y la posterior STC 31/2010, que avalaría este relato. Desde entonces, los catalanes se rigen por un Estatuto que solo parcialmente han votado y crece el apoyo a las posiciones independentistas, cuyas reivindicaciones, ahora sí, son «soberanistas».

No es posible en un trabajo como este abordar el sinfín de disputas jurídicopolíticas que suscita este proceso, ni siquiera las que puedan llegar a tener interés solo desde la visión de la teoría del Derecho. Por ello, concentraré mi atención en uno de esos ámbitos, que es el sentido y alcance jurídico que haya que dar al llamado «derecho a decidir». Salvo algunas pocas observaciones que añado ahora, he ido publicando a lo largo de los últimos años lo esencial de mi argumentación, tanto en trabajos académicos como en algún artículo periodístico ${ }^{1}$.

\section{Legalidad y legitimidad en el Estado constitucional contemporáneo}

Hay quien sostiene que las reivindicaciones provenientes de los partidarios del llamado «derecho a decidir» ni tienen cobertura legal ni son legítimas; los hay que consideran que, a pesar de que sean legítimas, no están amparadas por la legalidad; alguno habrá que entienda que, aunque fueran legales, carecerían de legitimidad; y, por último, hay quien opina que estas pretensiones pueden considerarse no únicamente legítimas, sino también ajustadas a la legalidad.

En lo que sigue voy a defender esta última opción. Empezaré mostrando qué significados pueden asociarse a los términos centrales de esta discusión, en especial

\footnotetext{
${ }^{1}$ Por lo que se refiere al ámbito académico, destacaré solo dos publicaciones: J.M. Vilajosana: "Principi democràtic i justificació constitucional del dret de decidir", Revista d'Estudis Autonòmics i Federals, n. 19, abril 2014, pp. 178-210 (Traducción castellana en el mismo número; versión inglesa: Josep M. Vilajosana. "The democratic principle and constitutional justification of the right to decide", Catalan Social Sciences Review, 4, 2014, pp 57-80) y J.M. Vilajosana: "Laboratorio Cataluña. Legalidad, legitimidad y eficacia en el proceso político catalán", en Iñaki Lasagabaster (coord.): Crisis institucional y democracia (A propósito de Cataluña). Valencia: Tirant lo Blanch, 2019, pp. 75-99. Por lo que hace al ámbito periodístico, puede verse J.M. Vilajosana: "Constitución, democracia y derecho a decidir", Eldiario.es, 4IV-2014 y J.M. Vilajosana: "La interpretación del derecho a decidir”, El País, 5-IV-2014.
} 
los de «legalidad» y «legitimidad». Una vez hecho esto, sostendré en qué sentido defiendo que el derecho a decidir en Cataluña es no solo legítimo sino también legal.

Por ahora bastará entender que la legalidad de un acto o de una norma es un rasgo relacional, según el cual un acto o una norma cumplen con la legalidad si se han llevado a cabo siguiendo lo establecido en un determinado sistema jurídico y, en última instancia, en su Constitución.

Sobre la legitimidad, cabe decir que pueden darse versiones muy distintas. Para lo que ahora interesa, se puede hablar de legitimidad en dos sentidos. Un primer concepto de legitimidad haría referencia al apoyo popular que reciba una determinada norma, una institución o el entero sistema jurídico. Podemos llamarla legitimidad formal. Es la que decimos que tiene un gobierno, por ejemplo, por el hecho de proceder de un parlamento escogido por sufragio universal. En este sentido, en el ámbito de la legitimidad formal tiene relevancia el juego de las mayorías, sea de votos o de representantes.

Por otro lado, se puede hablar de legitimidad de una norma, institución o sistema jurídico en un sentido substantivo, que sería el de no contravenir lo dispuesto en la moral. Ahora bien, al hablar de moral es preciso evitar caer en una confusión. Una cosa es la moral crítica, es decir, los valores y principios propios de una moral esclarecida, a la que se llega a través de un proceso de razonamiento, y otra la moral social o positiva, formada por los valores morales imperantes en una determinada sociedad en un período determinado. La razón de postular la existencia de una moral crítica es precisamente la de dar cuenta de la posibilidad de "criticar" las distintas morales sociales, realmente existentes.

Se puede ser escéptico frente a la existencia de la moral crítica, pero si se quiere ser consecuente con ese escepticismo, entonces o se cae en el relativismo moral (las únicas razones que pueden darse a favor o en contra de una institución son las que propias de la moral social de que se trate) o en el emotivismo (no cabe un reproche racional de cualquier moral social ya que la moral no sería una cuestión de razones, sino de emociones). Por el contrario, si creemos que es posible dar razones morales (y no solo expresar sentimientos de desaprobación) frente a prácticas como, por ejemplo, la de la mutilación genital femenina, y no nos parece justificado que se excluya del debate moral a alguien por el simple hecho de que esté en minoría respecto de los valores morales de la comunidad a la que pertenece, entonces hay que postular que existe otro nivel en el que se puede discutir racionalmente sobre materia moral. Ese nivel es el de la moral crítica.

Así, en este sentido substantivo, la referencia a la legitimidad de un acto o de una norma es ambigua. Para evitar esa ambigüedad, Garzón Valdés habló de legitimación y de legitimidad (Garzón Valdés, 1993). La legitimación de una norma o de un acto consistiría en su adecuación a la moral positiva, mientras que su legitimidad se relacionaría con la moral crítica. Aunque no siempre, tendencialmente la legitimación irá en la misma dirección que la legitimidad formal. Será así siempre que mayoritariamente los ciudadanos se comporten de manera racional y apoyen aquellas normas o instituciones que no contradicen sus valores morales. Sea como fuere, en lo que sigue entenderé por legitimidad substantiva únicamente la que se refiere a la moral crítica.

La existencia de la moral crítica permite censurar no solo una determinada moral positiva, sino también el Derecho positivo de una comunidad. Así, la legalidad de las normas de un sistema jurídico determinado y su legitimidad podrían distinguirse claramente. Ahora bien, si podemos hacer esto es porque tenemos, aunque sea de 
un modo intuitivo, alguna idea acerca de cuál sería el contenido de esa moral crítica. Eso es algo obviamente discutido desde hace siglos y las dificultades para ponerse de acuerdo son numerosas e importantes. Pero también hay que decir que se va produciendo de un tiempo a esta parte una cierta convergencia en considerar que el contenido y alcance de la moral crítica tiene que ver con el desarrollo de los derechos humanos.

\section{Derechos humanos y principios constitucionales}

Sin embargo, justamente la referencia a los derechos humanos ha sido y es la constante de las constituciones de la tradición democrático-liberal. Es una de las características que permite hablar de Estado constitucional de Derecho o de neoconstitucionalismo (Ferrajoli, 2007, vol. 1, pp. 822, 848; vol. 2, pp. 19-20, 62-63) ${ }^{2}$. La incorporación de un listado más o menos homogéneo de derechos fundamentales en las constituciones de carácter democrático ha hecho que se acabe sosteniendo tanto en el terreno doctrinal como en el jurisprudencial que el contenido de la constitución de alguna manera impregna todo el ordenamiento jurídico. Así, la visión neoconstitucionalista puede resumirse, por lo que importa, en dos rasgos ${ }^{3}$ :

a. La relevancia de los principios y no sólo de las reglas en la interpretación jurídica. Los derechos fundamentales están precisamente formulados en términos de principios y no de reglas.

b. Panconstitucionalismo: la omnipresencia de la Constitución en todos los ámbitos jurídicos, sin que quede ningún espacio significativo reservado en exclusiva a las leyes. Tal como lo expresa Prieto Sanchís, «detrás de todo precepto legal se vislumbra siempre una norma constitucional que lo confirma o lo contradice» (2001, p.208).

El hecho de que los derechos fundamentales se expresen mediante principios tiene dos implicaciones. En primer lugar, se precisará un plus de argumentación jurídica a la hora de determinar el contenido y el alcance de estos derechos. Al adoptar la forma de principios, estarán formulados en términos categóricos y no hipotéticos, por lo que, a diferencia de las reglas, carecerán de condiciones de aplicación o estas serán abiertas. Y ello comporta que muchas veces los principios deban introducirse en la argumentación jurídica a través de mecanismos como la ponderación y no mediante el clásico de la subsunción (Alexy, 1993, pp. 81-98). En segundo lugar, en la mayoría de ocasiones, los principios incorporan una carga político-moral. Por esta razón, no se puede establecer su contenido y alcance sin construir una teoría de este tipo, por rudimentaria que sea (Dworkin, 1984).

El segundo rasgo destacado implica que las constituciones son entendidas como verdaderas normas y no solo como programas políticos, por lo que su contenido permea todo el ordenamiento jurídico.

Si esto es así, es decir, si las constituciones incorporan los derechos humanos que hay que proteger y la legitimidad substantiva tiene que ver con el reconocimiento de tales derechos, entonces en puridad en un Estado constitucional de Derecho no cabe distinguir entre legalidad y legitimidad substancial de las normas cuando estas

\footnotetext{
2 La terminología aquí difiere.

${ }^{3}$ Reconstrucciones muy similares se hallan en Robert Alexy (1994). El concepto y la validez del Derecho. Barcelona, España: Gedisa, p. 159 y ss; G. Zagrebelsky (1995). El Derecho dúctil. Ley, derechos, justicia, Madrid, España: Trotta, p. 109 y ss.; R. Guastini (1998). "La 'costituzionalizzazione' dell'ordinamento italiano", Ragion Pratica, n. 11, p.185 y ss.; Prieto Sanchís (1999). Constitucionalismo y positivismo, México: Fontamara, $2^{\mathrm{a}}$ ed., 1999: 15 y ss.
} 
establecen derechos humanos. No podrá emitirse un juicio de legalidad (en puridad, de "constitucionalidad») sin uno previo de legitimidad (justamente el que hace referencia a los derechos humanos). Además, hay que tener presente que la regulación de tales derechos se hace a través de principios y no de reglas. El contenido y alcance de tales principios, por el tipo de materia que regulan, no pueden determinarse sin entrar antes en una discusión político-moral (Dworkin, 1984). Esa discusión será permanente y no puede darse por cerrada de una vez por todas en sociedades pluralistas como las nuestras (Rawls, 1996). Además, esa discusión será político-moral y relativa al contenido de la moral crítica, por lo que se debe apelar (y de hecho así suele hacerse) a razones de teoría política (por ejemplo, en el sentido de qué concepción de la democracia es mejor) y de teoría moral (por ejemplo, cuál es el alcance de la libertad, la igualdad, la dignidad). Esas apelaciones, sin embargo, no privan a las normas expresadas a través de los principios de su carácter jurídico. Es justo lo contrario: entrar en este tipo de consideraciones político-morales es lo exigible a la hora de interpretar tales principios jurídicos.

Por todo ello, en lo que sigue me ocuparé de postular una determinada interpretación de algunos principios reconocidos en la Constitución, tomando en cuenta cuestiones que afectarían, en mayor medida al ámbito de la legitimidad substantiva, pero sin olvidar otras referidas a la legitimidad formal (como en el apartado 5.2.2).

Mucho es lo que se ha escrito sobre los principios jurídicos, en general, y sobre los constitucionales, en particular ${ }^{4}$. En lo que sigue entenderé los principios constitucionales que entrarán en liza en este asunto como reglas ideales ${ }^{5}$.

Los principios, así concebidos, establecerían determinadas dimensiones de los estados de cosas ideales que el mundo debe tener para ser de conformidad con el Derecho. Es obvio que los distintos aspectos del ideal pueden entrar en conflicto entre sí, por ello, las reglas ideales deben ir acompañadas de mecanismos que establezcan el grado aceptable en que tales rasgos deben darse y eliminen los conflictos. Como es sabido, se lleva a cabo esta tarea a través de la técnica de la ponderación. Además, así como los principios a los que se adhiere una persona le confieren una determinada identidad, los principios jurídicos de un ordenamiento configuran su identidad material. Eso es lo que sucede con los principios constitucionales y de manera muy especial con los que nos encontraremos aquí: configuran la identidad de un sistema democrático liberal, entendido del modo que veremos posteriormente.

4. Derecho a decidir como derecho de la ciudadanía a ser consultada sobre su futuro político

Se ha dicho que el derecho a decidir es todavía un derecho en construcción ${ }^{6}$ (Bernadí, 2015, pp. 81-92). Su contenido y alcance vendrá determinado por el proceso de ponderación de los principios que están en juego. Pero, con el fin de dotarle de entidad propia, resulta conveniente distinguirlo del derecho a la autodeterminación ${ }^{7}$.

\footnotetext{
${ }^{4}$ Para un listado no exhaustivo de conceptos de principios jurídicos, véase Riccardo Guastini (1999). Distinguiendo. Estudios de teoría y metateoría del derecho. Barcelona, España: Gedisa, cap. 5.

${ }^{5}$ El concepto de regla ideal procede de Von Wright (1971), Norma y acción. Una investigación lógica. Madrid: Tecnos. Para su aplicación a los principios jurídicos, véase José Juan Moreso y Josep M. Vilajosana (2004), Introducción a la Teoría del Derecho. Madrid: Marcial Pons, pp. 68 y 91-92.

${ }^{6}$ Pero ya se ha avanzado bastante en esa construcción, como se pone de relieve a lo largo del texto.

${ }^{7}$ Ha subrayado tempranamente esta distinción, desde una perspectiva politológica, Jaume López (2011).

"Del dret a l'autodeterminació al dret a decidir", Quaderns de recerca, n. 4, pp. 2-18.
} 
Podríamos resumir en tres sus respectivos rasgos distintivos:

a. El derecho a decidir es un derecho individual (cuyos titulares son cada uno de los ciudadanos y ciudadanas de un determinado demos), aunque se ejerza colectivamente; en cambio, el derecho a la autodeterminación es un derecho colectivo (cuyos titulares son los pueblos).

b. Los derechos en cada caso se vinculan a dos diferentes formas típicas de ejercicio (aunque no necesariamente excluyentes). En el primer caso, prima la realización de una consulta, que requiere alguna acción u omisión por parte del Estado (autorizándola, delegando su competencia o, simplemente, tolerándola); en el segundo, se opta por la declaración unilateral de independencia.

c. La legalidad tomada como referencia en ambos casos es distinta. En el supuesto del derecho a decidir, sería el sistema jurídico estatal, mientras que en el caso del derecho a la autodeterminación sería el Derecho internacional.

A continuación, me ocuparé solo del derecho a decidir, entendido como el que tiene la ciudadanía «de una comunidad territorialmente localizada y democráticamente organizada (en este caso, la catalana) a expresar y realizar, mediante un procedimiento democrático, la voluntad de redefinir el estatus político y marco institucional fundamentales de dicha comunidad, incluida la posibilidad de constituir un Estado independiente» (Barceló et al., 2015, p. 13).

Con el fin de especificar el alcance jurídico de esta cuestión, es mejor formularla teniendo en cuenta que actualmente existen unas instituciones representativas de los ciudadanos de una Comunidad Autónoma, en este caso Cataluña. Así, la pregunta práctica es: ¿la Constitución española permite que la Generalitat plantee una consulta a los catalanes sobre su futuro político? Las ventajas de formular el asunto en estos términos son que conecta al titular del derecho (la ciudadanía) con la forma típica de ejercerlo institucionalmente (la consulta) y que hace aflorar la ambivalencia que quedaría encubierta si la cuestión la planteamos simplemente en términos de derechos de los ciudadanos. Me refiero a la típica ambigüedad de los permisos.

Permitir una conducta en sentido débil es equivalente a afirmar que, en el sistema jurídico de que se trate, no hay una norma que prohíba esta conducta. En cambio, cuando alguien sostiene que una conducta está permitida en sentido fuerte, lo que dice es que en el sistema jurídico relevante hay una norma de permisión. Por lo tanto, en el primer caso, se trata de mostrar la ausencia de una norma (de prohibición), mientras que, en el segundo, hay que mostrar la existencia de una norma (de permisión) (Von Wright, 1971, p.13). Como comprobaremos en este trabajo, hay buenos argumentos para pensar que la Constitución permite, al menos en sentido débil, realizar una consulta a la ciudadanía de Cataluña sobre su futuro político ${ }^{8}$.

\section{La ponderación de los principios relevantes}

Lo primero que hay que decir es que no hay ninguna regla en la Constitución que regule el caso planteado por la pregunta. Por ello hay que acudir a los principios constitucionales. Quien se opone a una consulta que trate sobre la independencia de

\footnotetext{
${ }^{8}$ Lo cual no excluye, por supuesto, que quepa argumentar de manera plausible que se da un permiso fuerte. Al respecto, véase Mercè Barceló, 2015, op. cit. pp. 109-112.
} 
Cataluña esgrime básicamente dos principios. El primero, contenido en el art. 2 CE, el de la indisoluble unidad de la nación española; el segundo, el de la soberanía del pueblo español, recogido en el art. 1.2. CE. El principio que habría que ponderar frente a estos es el principio democrático establecido como pórtico en el art. 1.1. CE y concretado después en otros, como el de participación política del art. $23.1 \mathrm{CE}$. A continuación, expondré brevemente, en el orden mencionado, una manera de ponderar esos principios, que me parece razonable y a la altura de lo que exige una democracia de calidad en el siglo XXI.

\subsection{El principio democrático como límite al principio de indisolubilidad}

Los contrarios a la justificación constitucional de la consulta articulan su interpretación del principio de indisolubilidad de la nación española a través de sendos argumentos que denominaré, respectivamente, el argumento de la primacía absoluta del principio de indisolubilidad y el argumento de la petrificación del principio democrático ${ }^{9}$. Respecto del primero, sostendré que, como todos los principios, también el de indisolubilidad tiene límites; con respecto al segundo, me opondré defendiendo una interpretación evolutiva de la Constitución y, en concreto, de los derechos que directamente están vinculados con el principio democrático.

El argumento de la primacía absoluta del principio de indisolubilidad defendería que no hay ninguna circunstancia concebible en que el principio democrático pueda prevalecer. Una consulta sobre la independencia de una parte del territorio estatal afecta al principio de indisolubilidad (admitamos que lo afecta tendencialmente, pues no alcanzo a comprender cómo el hecho de celebrar un referéndum por sí solo destruye la unidad territorial). Por lo tanto, concluiría el argumento, una consulta de este tipo no puede recibir cobijo bajo el principio democrático.

Este razonamiento, sin embargo, resulta chocante. Hasta ahora se había admitido, tanto por parte de la doctrina como por la jurisprudencia del Tribunal Constitucional, que la herramienta de la ponderación entre principios servía precisamente para no entender ninguno de ellos de forma absoluta. En determinadas circunstancias, prevalecería uno; en circunstancias distintas, prevalecería el otro. Por las razones que después aduciré, creo que, en este caso, debería prevalecer el principio democrático.

En cualquier caso, para ser consecuente, es necesario que añada los casos en que el principio de indisolubilidad prevalece sobre el democrático. Es una cuestión pacífica que la Constitución no reconoce un derecho a la autodeterminación. Por lo tanto, la declaración unilateral de independencia por parte de la Generalitat, como el ejemplo típico de ejercicio de aquel derecho, no tendría cobertura constitucional, aunque la hagan los representantes democráticamente escogidos ${ }^{10}$. De este modo, ninguno de estos dos principios sería entendido de manera ilimitada y ambos

\footnotetext{
${ }^{9}$ A pesar de lo que digo en el texto, no es fácil encontrar un desarrollo explícito de estos argumentos. Quienes niegan que el derecho a decidir de los catalanes quepa en la Constitución se limitan a citar el artículo sin aportar razones adicionales, como si el principio democrático fuera en este caso irrelevante. Por eso, los argumentos que intento reconstruir suelen hallase en el debate solo de manera implícita. Tampoco las diversas sentencias del TC han hecho, a mi modo de ver, una ponderación explícita y argumentada de esos principios. La que más se ha acercado a ello es la STC 42/2014, de 25 de marzo de 2014 , en la que por primera vez se menciona la expresión «derecho a decidir», se la contrapone al derecho a la autodeterminación (como aquí he hecho) y se cita el Dictamen de la Corte Suprema de Canadá de1998. A pesar de ello, insiste en que la aspiración del «derecho a decidir» solo cabe como pretensión de reforma constitucional, con lo que en realidad se aparta de lo que dice ese Dictamen, como después veremos.

${ }^{10}$ Como, por otra parte, sostiene también el mencionado Dictamen de la Corte Suprema canadiense.
} 
intervendrían en la configuración de la identidad de la Constitución de manera ponderada.

\subsubsection{Una interpretación evolutiva de los derechos democráticos}

El argumento de la petrificación de la Constitución parte de la premisa de que si era necesaria una ponderación entre el principio de indisolubilidad y el principio democrático, ya la hizo en su momento el legislador constituyente. Así, la plasmación del principio democrático en el artículo 1.1, dentro de los que el Tribunal Constitucional ha denominado «principios estructurales» admitiría una y solo una estructuración, una y solo una realización concreta: la plasmada en el resto del articulado de la Constitución. Como no se contempla expresamente este tipo de consulta, se diría entonces que la forma en que el constituyente entendió la democracia es ésta y no puede ser alterada, salvo modificación expresa del texto constitucional.

Podemos aceptar que, en el momento de ser aprobado el texto constitucional, no estuviera presente entre la mayoría de los miembros de las Cortes constituyentes la idea de que la Constitución amparaba una consulta como la que ahora se plantea. También podemos admitir que no se hubiera previsto que los instrumentos de democracia directa reconocidos en la Constitución, como el referéndum, pudieran ser utilizados de una forma más activa de la que en aquel momento se pensaba, vistas las lógicas prevenciones frente a su empleo por parte del régimen franquista. Pero del hecho de que en aquel momento no existiera esta percepción, no podemos inferir que la Constitución interpretada cuatro décadas después no pueda ir más allá. Esta adecuación a las concepciones cambiantes es precisamente lo que facilita la interpretación evolutiva.

El uso de la interpretación evolutiva goza de una larga tradición en la jurisdicción internacional y, más en concreto, en la del Tribunal Europeo de Derechos Humanos a la hora de interpretar el Convenio de Derecho Humanos (Prebensen, 2000, pp. 1123-1138). También se ha utilizado, entre otros, por el Tribunal Internacional de Justicia ${ }^{11}$ por el Tribunal Europeo de Justicia ${ }^{12}$, por la Corte interamericana de Derechos Humanos ${ }^{13}$ o por el Comité de Derechos Humanos de las Naciones Unidas ${ }^{14}$.

También el Tribunal Constitucional ha utilizado la interpretación evolutiva en alguna ocasión. En la Sentencia 198/2012 de 6 de noviembre, la emplea para demonstrar el alejamiento de la voluntad de los constituyentes respecto a la institución matrimonial, admitiendo que la modificación del Código Civil, que permite el matrimonio entre personas del mismo sexo, es constitucional. Y lo justifica así: «el Tribunal Constitucional, cuando controla el ajuste constitucional de esas actualizaciones, dota a las normas de un contenido que permite leer el texto constitucional a la luz de los problemas contemporáneos, y de las exigencias de la

\footnotetext{
${ }^{11}$ Algunos de los casos donde se puede hallar son: Legal Consequences for States of the Continued Presence of South Africa in Namibia (South West Africa) notwithstanding Security Council Resolution 276 (1970), [1971] ICJ Reports 16, para. 53. Aegean Sea Continental Shelf [1978] ICJ Reports 3, para. 77. Dispute regarding Navigational and Related Rights (Costa Rica v. Nicaragua) [2009] ICJ Reports 213, para. 64-66.

12 Caso 283/81, SrI CILFIT and Lanificio di Gavardo SpA v Ministry of Health [1982] ECR 3415, para. 20.

${ }^{13}$ The Right to Information on Consular Assistance in the Framework of the Guarantees of the due Process of Law, Advisory Opinion, OC-16, Inter-American Court of Human Rights Series A No 16 (1 October 1999), para. 114.

${ }^{14}$ Roger Judge v. Canada, Human Rights Committee, Communication No. 829/1998, U.N. Doc. CCPR/C/78/D/829/1998 (2003), para. 10.3.
} 
sociedad actual a que debe dar respuesta la norma fundamental del ordenamiento jurídico a riesgo, en caso contrario, de convertirse en letra muerta» (fundamento $9^{\circ}$ ).

Dado el papel fundamental asignado a la interpretación evolutiva tanto en la ya consolidada jurisprudencia internacional como en la más incipiente del Tribunal Constitucional, queda la translación a nuestro caso. Para ello, deberemos ser sensibles a la evolución que ha sufrido la institución de la democracia en los últimos tiempos y ser capaces de aplicar los resultados que obtengamos a los derechos y libertades que están claramente asociados con ella. No se debe olvidar a lo largo de este camino el mandato interpretativo del artículo 10.2, tal como el mismo TC lo ha interpretado ${ }^{15}$. Lo que le lleva a sostener que «la regla hermenéutica del art. 10.2 CE lleva asociada una regla de interpretación evolutiva» (fundamento $8^{\circ}$ ). Por esta razón, propongo una concepción «densa» de la democracia que encaja perfectamente con la interpretación evolutiva y de la que podremos inferir más adelante consecuencias relevantes con el fin de interpretar los derechos y libertades que se vinculan con ella (emblemáticamente, el derecho fundamental reconocido en el art. 23.1), así como los fundamentos de la identidad del sistema democrático liberal, (dignidad humana y libre desarrollo de la personalidad que encontramos en el art.10.1).

\subsubsection{Una concepción densa de la democracia}

La metáfora de la densidad facilita visualizar una idea dinámica de la democracia, como conjunto de estratos superpuestos, pero que no son fruto de una simple acumulación, sino que constituyen una integración coherente y nunca acabada. Por lo que ahora interesa, esta concepción se concretaría en dos vertientes, una procedimental y otra de contenido.

Con respecto a la vertiente procedimental, una concepción densa de la democracia nos ayuda a entender que no tiene sentido plantear, como a veces se hace precisamente para negar la posibilidad de justificar la consulta, que hay dos sistemas democráticos irreconciliables: el representativo y el directo. Según esta línea argumental que critico, la Constitución habría consagrado un modelo de democracia representativa, en el que cualquier elemento de democracia directa sería visto con aprensión y por lo tanto limitado a su mínima expresión.

A veces parece como si quisiera decirse que convocar referéndums es antidemocrático, olvidando que lo que hace que éste sea un instrumento verdaderamente democrático no es el mecanismo en sí, sino el contexto institucional en que se enmarca. Los referéndums de la época franquista eran antidemocráticos, no porque fueran referéndums, sino porque se desarrollaban dentro de un régimen autoritario. Los que se pueden convocar ahora, en cambio, son plenamente democráticos porque pueden materializarse en un entorno pleno de garantías electorales y de respeto por los derechos fundamentales. Las elecciones y las consultas pueden convivir perfectamente, sin que el uso de un procedimiento excluya al otro. Esta coexistencia, lejos de desnaturalizar una supuesta esencia representativa inmutable presente en la Constitución, enriquece sus componentes, haciéndola de más calidad, más "densa". Y eso es perfectamente compatible con el derecho fundamental que los ciudadanos tienen de participar directamente en los asuntos públicos, tal como se reconoce en el artículo 23.1 de la Constitución.

\footnotetext{
${ }^{15}$ Entre otras, en las STC 116/2006, de 24 de abril y STC 38/2011, de 28 de marzo. Así, es en el art. 10.2 CE donde encuentra sustento la interpretación evolutiva según el TC, y no en el art. 3.1. del Código Civil.
} 
Esta densidad de la democracia también se manifiesta en su contenido. Las democracias actuales son fruto de sucesivas conquistas en los derechos de las personas y de los colectivos de los que forman parte. La sensibilidad que hoy se tiene, por ejemplo, con respecto a la importancia que han ido adquiriendo los llamados derechos de tercera generación, (que incluyen entre otros los derechos a la autodeterminación y a la identidad nacional) se puede apreciar no tan sólo en la teoría política contemporánea (Ishay, 2008), sino también en la visión de organismos internacionales ${ }^{16}$. Hace tiempo que el desarrollo de la democracia pasa por ampliar las esferas en que los ciudadanos puedan decidir democráticamente. Así lo expresaba Bobbio hace más de treinta años: «Hoy quien quiera tener un indicador del desarrollo democrático de un país, ya no debe considerar el número de las personas que tiene derecho al voto, sino el número de lugares diferentes de los tradicionalmente políticos en los que se ejerce el derecho al voto» (Bobbio, 1985, p. 220).

Lo que quiero destacar de este fenómeno no es sólo que sea una visión nueva, sino que es perfectamente integrable en las anteriores. La perspectiva de las democracias liberales está mudando, pero no para decir que estos nuevos derechos no encajan. Precisamente, cambia para facilitar su encaje. El comportamiento del Reino Unido respecto de las demandas escocesas o el de Canadá en relación con las de Quebec, son buena prueba de ello. Es inexcusable mencionar en este punto el Dictamen de la Corte Suprema canadiense de 1998: «Le rejet clairement exprimé par le peuple du Québec de l'ordre constitutionnel existant conférerait clairement légitimité aux revendications sécessionnistes, et imposerait aux autres provinces et au gouvernement fédéral l'obligation de prendre en considération et de respecter cette expression de la volonté démocratique en engageant des négociations et en les poursuivant en conformité avec les principes constitutionnels» (Renvoi relatif à la sécession du Québec, de 20-I-1998, ap. 88) ${ }^{17}$.

La concepción densa de la democracia, en definitiva, sirve para dotar de contenido a la interpretación evolutiva que esta institución requiere, tal como he explicado. Se sigue de todo lo que he sostenido en este apartado que la interpretación ponderada del principio democrático (concretado en el artículo 23.1) con el de indisolubilidad es tal que permite (cuando menos, en sentido débil) la convocatoria de un referéndum sobre la independencia de Cataluña, pero veta una declaración unilateral. Ésta es una forma razonable de tomar estos principios como reglas ideales y definitorios del Estado democrático instaurado en el artículo 1.1 de la Constitución.

Debo añadir, además, que esa discusión acerca del contenido y el alcance del principio democrático debería llevarse a cabo en el nivel de la moral crítica, como he dicho. En este sentido, me parece que en la mencionada STC 148/2012 el alto tribunal toma la evolución de la que habla al aplicar la interpretación evolutiva como una constatación empírica de un supuesto cambio en los valores dominantes de la sociedad. Pero este proceder tiene algún inconveniente, a mi modo de ver. Implica pensar que esta discusión es una cuestión relativa a la moral social, cuando en realidad debería relacionarse con la moral crítica. El argumento del TC hubiera tenido que ir en la línea de ofrecer buenas razones para ampliar la posibilidad de contraer matrimonio a las personas del mismo sexo. Y esas buenas razones, por ejemplo, pasaban por demostrar que esa ampliación es más acorde con el principio de igualdad y no discriminación por razones de orientación sexual (art. 32 en relación con el art. $14 \mathrm{CE})$. Hacer eso supone entrar ya en una discusión relativa a la moral crítica. No es

\footnotetext{
${ }^{16}$ Cfr., como ejemplo, Human Development Report, Naciones Unidas, 2004, en el que se sostiene que las libertades culturales y nacionales son componentes importantes para la calidad de la democracia.

${ }^{17}$ Cabe recordar que tal pronunciamiento tiene lugar después de que se hayan realizado en Quebec dos referéndums (en 1980 y 1995), que, si bien no fueron acordados como el de Escocia en 2014, sí al menos fueron "tolerados".
} 
una mera constatación de qué valores son los imperantes en ese momento en la sociedad, es decir, de cuál es su moral social. Si fuera así, en el momento que cambiaran las mayorías en España, por ejemplo, dejaría de ser discriminatorio por razón de orientación sexual que se prohibiera que las personas del mismo sexo contrajeran matrimonio ${ }^{18}$. Así pues, la evolución de la que se debería hablar al usar la interpretación evolutiva, al menos como la defiendo aquí, es la que tiene lugar en relación con la mejor concepción político-moral que se posea en el momento. Esta, por supuesto, siempre es falible y puede evolucionar en el futuro, pero no tiene nada qué ver con el número de personas que la sostienen, sino con la calidad de sus $\operatorname{argumentos}^{19}$.

\subsection{El principio democrático como límite al principio de la soberanía nacional}

A pesar de haber razonado que la Constitución permite la consulta, falta discutir a quién se debe consultar: ¿sólo a los catalanes o a todos los españoles? De nuevo encontramos diferentes principios constitucionales en disputa. Para quien cree que el sujeto de la consulta no puede ser la ciudadanía catalana, sino que debe ser la española, el fundamento sería el principio según el cual la soberanía reside en el pueblo español (art. 1.2.). Sin embargo, ese principio también tiene límites. Estos límites surgen de nuevo de la ponderación con el principio democrático. Pero antes de concretar esa ponderación es conveniente salir al paso de una crítica que tiene que ver con las dificultades para determinar el demos.

\subsubsection{La determinación del demos}

Una de las objeciones recurrentes de los contrarios al derecho a decidir es que hay dificultades para la determinación del demos, es decir, de cuál es el colectivo relevante a la hora de ser consultado. El argumento suele adoptar la forma de reducción al absurdo. Así, se dirá que si los catalanes tienen ese derecho, qué impide que lo tengan los habitantes del área metropolitana de Barcelona; y si estos lo tienen, por qué no los de una población determinada dentro de esa área. Dicho en términos generales, nada habría que justificase que una clase determinada de individuos sea la relevante para ser la titular de ese derecho, ya que podría serlo cualquier subclase de la misma. Se sobreentiende que la conclusión a la que podría hacerse llegar a los defensores del derecho a decidir de los catalanes sería absurda porque con igual legitimidad (o con tan poca) podrían esgrimir tal pretensión, pongamos por caso, los habitantes de Terrassa.

Frente a este argumento cabe oponer lo siguiente. Para empezar, o el argumento es plausible o no lo es. Si no lo es, es decir, si no se considera que la conclusión es absurda, no hace falta que le dediquemos más tiempo. $\mathrm{Si}$, en cambio, se supone que es plausible, entonces habrá que admitir que se aplica no solo a la clase de los catalanes sino a cualquier otra clase de ciudadanos. Así, la misma reducción al absurdo se produciría respecto a España. ¿Por qué los españoles pueden decidir su futuro político y no cualquier subclase de ciudadanos, entre los cuales por cierto la de los catalanes? Ante este contraargumento, ya solo queda refugiarse en la defensa de lo existente. Ha habido a lo largo del tiempo distintas versiones de esta tesis. Desde equiparar lo real a lo racional, a la Hegel, hasta sostener que ya hemos alcanzado el final de la Historia, por decirlo en términos de Fukuyama. Pero, llegados a este punto, o bien se cae en el problema de la inducción,

\footnotetext{
${ }_{18}$ De hecho, esta posibilidad parece ser admitida implícitamente en la propia STC, al aceptar que el legislador podría regular el matrimonio de manera distinta y ello no vulneraría el art. 32CE.

${ }^{19}$ En la línea defendida en numerosos trabajos por Ronald Dworkin. Véase, por todos, Ronald Dworkin, Los derechos en serio, op. cit.
} 
al pensar que porque algo es de una determinada manera lo seguirá siendo en el futuro (con multitud de contraejemplos históricos que ponen de relieve que el número de Estados en el mundo ha sido de todo menos estable), o bien en la falacia naturalista, al dar valor normativo a lo que no es más que una cuestión de hecho.

\subsubsection{Principio de la mayoría y dominio de la mayoría}

Visto lo anterior, es momento de entrar en la ponderación. Empezaré planteando dos hipótesis. La primera es muy simple: imaginemos que en Cataluña la totalidad del cuerpo electoral quisiera votar «sí» en un referéndum sobre la independencia. Este $100 \%$ de catalanes seguiría siendo una minoría en España. Por lo tanto, es previsible que perdiera sistemáticamente cualquier votación sobre esta cuestión que tuviera como sujeto a todos los españoles. Ahora bien, preguntémonos no ya cómo se sentirían los catalanes (la respuesta es obvia), sino cómo debería sentirse en una situación como ésta alguien que pertenezca a la perpetua mayoría. ¿Podría seguir diciendo que vive en un Estado democrático y liberal? ${ }^{20}$

La segunda hipótesis es algo más compleja, pero sus implicaciones son aún más diáfanas que en el caso anterior ${ }^{21}$. Si dividimos los votantes de un eventual referéndum sobre la independencia de Cataluña entre los ciudadanos de ésta y los de (el resto de) España, normalmente pensamos en la dirección expresada en la primera hipótesis: gana el sí en Cataluña (aunque no obviamente con el 100\% de los votos), y el no en España. Por supuesto, dos de las otras posibilidades no presentan problemas, como sería que el resultado de la consulta sea coincidente en uno y otro colectivo (ambos votan sí, o ambos votan no). Pero la lógica nos indica que cabe una cuarta posibilidad, por improbable que esta sea: que gane el no en Cataluña y el sí en España. ¿Sería esta una buena razón para que la mayoría de españoles expulse Cataluña de España, en contra de la voluntad de la mayoría de los catalanes? No parece razonable esta conclusión. Pero, si no lo es, entonces el colectivo relevante es el de los catalanes ${ }^{22}$.

Experimentos mentales como estos nos ponen sobre la pista de que hay algo que no encaja en la defensa de que el sujeto relevante de este tipo de consultas sea la totalidad del pueblo español. ¿O se da el caso de que esa opinión mayoritaria valdría solo para vetar la independencia, pero no para validar la expulsión?

Lo que no encaja es esto: la democracia implica el principio de la mayoría, pero es contraria al dominio de la mayoría sobre la minoría, en este caso una minoría con contornos territoriales definidos y con lengua, cultura e instituciones propias ${ }^{23}$. El dominio de la mayoría pervierte la democracia y se opone a los principios definitorios de los Estados liberales. Hagamos un breve repaso de estos principios; después veremos que ocupan un lugar destacado en los Tratados de la Unión Europea y en la

\footnotetext{
20 Utilizo el adjetivo «liberal» como contrapuesto a «fundamentalista», no como contrario a «social». Para un análisis más pormenorizado de los principios definitorios de una sociedad liberal, remito a Josep M. Vilajosana (2017). Identificación y justificación del Derecho. Madrid: Marcial Pons, $2^{\text {a }}$ ed., cap. 6.

${ }^{21}$ La formulación de esta hipótesis se la escuché al filósofo Josep Maria Terricabras.

22 Por otro lado, así se ha entendido en la larga lista de referéndums realizados en el mundo desde el de Liberia en 1846 hasta el de Nueva Caledonia, celebrado el 4 de noviembre de 2018.

${ }^{23}$ Como es sabido, en teoría política Tocqueville habló de la tiranía de la mayoría. En el ámbito de la teoría del Derecho, la distinción entre principio y dominio de la mayoría se encuentra en Kelsen, si bien este autor no había pensado obviamente en aplicarla a las minorías territorializadas. Pero si tenemos en cuenta la concepción densa de la democracia que defiendo en el texto, entonces no hay ningún impedimento para hacerlo. Cfr. Hans Kelsen (1954). Teoría general del Estado. México: Editora Nacional, (original de 1925), pp. 412-413.
} 
Constitución. Son los principios de autonomía, inviolabilidad y dignidad de la persona (Nino, 1989).

\subsubsection{Los principios de autonomía, inviolabilidad y dignidad de la persona}

Según el principio de autonomía de la persona, los individuos deben ser responsables por la elección de sus planes de vida de acuerdo con sus preferencias, y no ver esta elección como un hecho del que son víctimas. Se trata de un principio que permite justificar los bienes sobre los que versan ciertos derechos fundamentales en nuestras sociedades contemporáneas. Estos bienes son los indispensables para la elección y mantenimiento de los planes de vida que los individuos puedan proponerse. Entre estos derechos estarían los de participación política como el reconocido en el art. 23 CE. La aplicación a nuestro caso: si para un amplio número de catalanes cuenta mucho para el desarrollo de sus planes de vida ser consultados sobre su futuro político, hasta el punto de incluir en la consulta la posibilidad de constituir una entidad política independiente, no pueden ser ignorados arguyendo simplemente que son una minoría dentro del Estado. ${ }^{24}$

El principio de inviolabilidad de la persona consiste en sostener que no se pueden imponer a las personas contra su propia voluntad sacrificios y privaciones que no redunden en su propio beneficio. Recuérdese en este sentido la segunda formulación del imperativo kantiano: «Actúa de manera tal que nunca trates a la humanidad, sea en tu propia persona o en la de los otros, como un mero medio sino siempre como un fin en sí mismo» (Kant, 1983). La idea de no instrumentalizar a las personas para obtener otras finalidades ajenas a su bienestar es la que fundamenta claramente el veto al dominio de la mayoría. Si se produce este dominio, que además se admite sin rubor que pueda serlo de manera perpetua, se está instrumentalizando a las personas que pertenecen a la minoría, se las trata como meros destinatarios de las políticas de otros y no como sujetos, cuyas preferencias hay que tener en cuenta.

Por último, tratar a las personas con la dignidad que merecen supone tomarse seriamente sus creencias, sus opiniones, pero también sus decisiones. Tomarse en serio las decisiones de un individuo, consiste, entre otras cosas, en permitirle que asuma las consecuencias de las mismas, que las incorpore al curso de su vida. Se trata de tomar decisiones entre personas con igual dignidad, sin que importen las razones por las que acaben decidiendo: el voto legitima con independencia de las razones que cada uno tenga para emitirlo25.

Lo dicho no es solo «filosofía». Es, como he sostenido aquí, el modo de dotar de contenido, con razonamientos político-morales, a los principios constitucionales, ergo jurídicos, y que, además, conforman la identidad de un Estado democráticoliberal. Tal como se establece en el art. 9 («Corresponde a los poderes políticos promover las condiciones a fin de que la libertad (...) del individuo y de los grupos donde se integra sean reales y efectivas») y en el art. 10 («La dignidad de la persona (...), el libre desarrollo de la personalidad (...) son fundamento del orden político y de la paz social»). ${ }^{26} \mathrm{~A}$ lo anterior hay que añadir que los Tratados de la Unión europea

\footnotetext{
${ }^{24}$ Las encuestas con las que se cuenta desde hace años arrojan un balance claro. En todas ellas, entre el $70 \%$ y el $80 \%$ de los ciudadanos residentes en Cataluña estarían de acuerdo con la celebración de un referéndum acordado para decidir su futuro político.

${ }^{25}$ Sobre la dignidad humana como fundamento de la democracia, véase Ronald Dworkin (2008). La democracia posible. Principios para un nuevo debate político. Barcelona, España: Paidós.

${ }^{26}$ Sobre la relevancia de este artículo, se ha dicho que «sin hipérbole puede estimarse que este artículo de nuestra Magna Carta es piedra angular de todo el sistema jurídico que ella instituye», en Joaquín Ruiz-Giménez Cortés y Itziar Ruiz-Giménez Arrieta, "Comentario al Artículo 10 de la Constitución Española", en Oscar Alzaga Villaamil, Santiago Sánchez González, et al. (dir.), Comentarios a la Constitución Española. Tomo II - Artículos 10 a 23 de la Constitución Española de 1978, Edersa, 2006.
} 
los reconocen explícitamente, relacionándolos con el respeto de las minorías. Así, en el art. 2: «La Unión se fundamenta en los valores del respeto a la dignidad humana, de la libertad, de la democracia (...) y del respeto a los derechos humanos, incluidos los derechos de las personas pertenecientes a minorías».

\subsubsection{Alcance ponderado de la soberanía nacional}

Antes de concluir, quedaría por examinar cuál sería la ponderación de los principios que conforman una democracia liberal como la que se define en la Constitución con el principio de la soberanía nacional. Después de lo que acabo de decir, debe quedar claro que el peso de este último principio no es suficiente para impedir una consulta, pero justifica que los ciudadanos del resto del Estado tengan que intervenir de alguna forma.

Esta intervención puede ser desempeñada por los representantes de la ciudadanía española en el Parlamento. Ello se consigue aceptando cualquiera de las vías a través de las cuales cabe articular constitucionalmente el derecho a decidir ${ }^{27}$. En primer lugar, de manera directa, según lo dispuesto en el artículo 92. En este caso se permite que las decisiones políticas de especial transcendencia sean sometidas a referéndum, convocado por el rey, mediante propuesta del gobierno y con autorización del Congreso de los diputados. En segundo lugar, a tenor de lo dispuesto en el artículo 150.2, el Estado podría transferir o delegar la facultad de convocar un referéndum a la comunidad autónoma. Ambas vías, pues, comportan la intervención decisiva de los representantes del conjunto de los ciudadanos del Estado, a través de los cuales los términos del referéndum podrían ser objeto de negociación (fecha, pregunta, quórum, efectos, etc. $)^{28}$.

Hasta aquí, pues, una propuesta que intenta armonizar, creo que de manera razonable, los principios que confieren identidad a un Estado democrático y de derecho como el consagrado en la Constitución de 1978 leída cuatro décadas después. No pretendo dar a entender que sea la única manera de hacerlo ${ }^{29}$. Pero, en todo caso, quien se oponga a este o a otros intentos parecidos de encontrar una salida respetuosa con el principio democrático se encontrará con que sigue en pie la pregunta inicial que ha originado este trabajo y que, ya parar terminar, podemos reformular así: ¿En pleno siglo XXI habrá que seguir pensando que la única posibilidad de crear nuevos Estados estriba en la utilización de medios violentos como ha sido tradicional en el pasado o, por el contrario, los sistemas democráticos pueden y deben tener una respuesta distinta que pase por articular las demandas en este sentido de una forma ordenada, pacífica y respetuosa con la voluntad de los gobernados como la que supone la celebración de un referéndum?

\footnotetext{
${ }^{27}$ Sobre las distintas vías, véase las que ya se analizaban en el «Informe sobre els procediments legals a través dels quals els ciutadans i les ciutadanes poden ser consultats sobre llur futur polític», elaborado por el Institut d'Estudis Autonòmics, de 11 de marzo de 2013.

${ }^{28}$ Sobre los problemas que debería sortear un referéndum de estas características remito de nuevo al citado Dictamen de la Corte Suprema canadiense. Un buen análisis teórico reciente de ellas, se encuentra en Pau Bossacoma. Morality and Legality of Secession. A Theory of National SelfDetermination. Palgrave Macmillan, 2020, cap. 11.

${ }^{29}$ Véanse otras, por ejemplo, en Jorge Cagiao y Gennaro Ferraiuolo (coords.). El encaje constitucional del derecho a decidir. Un enfoque polémico. Madrid: Catarata, 2016.
} 
Bibliografía

Alexy, R. (1993). Teoría de los derechos fundamentales. Madrid, España: Centro de Estudios Constitucionales.

Alexy, R. (1994). El concepto y la validez del Derecho. Barcelona, España: Gedisa.

Barceló, M., Corretja, M., González, A., López, J.; Vilajosana, J. M. (2015). El derecho a decidir. Teoría y práctica de un nuevo derecho. Barcelona, España: Atelier, 2015.

Bernadí, X. (2015). El derecho a decidir: características básicas de un derecho en construcción", en M. Barceló y C. Luiz (coord.). Escritos sobre derechos individuales y colectivos. Una visión desde perspectivas constitucionales diversas (pp. 81-92). Barcelona, España: Atelier.

Bobbio, N. (1985). Estado, gobierno y sociedad: Por una teoría general de la política. México: FCE.

Bossacoma, P. (2020). Morality and Legality of Secession. A Theory of National SelfDetermination. Palgrave Macmillan.

Cagiao, J. y Ferraiuolo, G. (coords.) (2016). El encaje constitucional del derecho a decidir. Un enfoque polémico. Madrid, España: Catarata.

Dworkin, R. (1984). Los derechos en serio. Barcelona, España: Ariel.

Dworkin, R. (2008) La democracia posible. Principios para un nuevo debate político. Barcelona, España: Paidós.

Ferrajoli, L. (2007). Principia iuris. Teoria del diritto e della democrazia. Roma-Bari, Italia: Laterza.

Garzón Valdés, E. (1993). El concepto de legitimidad en Niklas Luhmann. Derecho, ética, política (pp. 509-517), Madrid, España: Centro de Estudios Constitucionales.

Guastini, R. (1998) "La 'costituzionalizzazione' dell'ordinamento italiano", Ragion Pratica, n. 11, pp 185-206.

Guastini, R. (1999). Distinguiendo. Estudios de teoría y metateoría del derecho. Barcelona, España: Gedisa.

Ishay, M. (2008). The History of Human Rights: From Ancient Times to the Globalization Era. Berkeley, Estados Unidos: University of California Press, 2008.

Kant, I. (1983). Fundamentación de la metafísica de las costumbres. Madrid, España: Espasa Calpe. (original de 1785).

Kelsen, H. (1954 [1925]). Teoría general del Estado. México: Editora Nacional.

López, J. (2011). Del dret a l'autodeterminació al dret a decidir. Quaderns de recerca, n. 4 , pp. 2-18.

Moreso, J. J. y Vilajosana, J. M. (2004). Introducción a la Teoría del Derecho. Madrid, España: Marcial Pons.

Nino, C. S. (1989). Ética y derechos humanos. Un ensayo de fundamentación. Barcelona, España: Ariel.

Prebensen, S. C. (2000). Evolutive interpretation of the European Convention on Human Rights. P. Mahoney, F. Matscher, H. Petzold \& L. Wildhaber (eds.), Protecting Human Rights: The European Perspective - Studies in memory of Rolv Ryssdal (pp. 1123-1138), Alemania, Colonia: Carl Heymanns Verlag.

Prieto, L. (1999). Constitucionalismo y positivismo. México: Fontamara.

Prieto, L. (2001). Neoconstitucionalismo y ponderación judicial, AFDUAM n. 5, 2001, pp. 201-228.

Requejo, F. (2010) Camins de democràcia. De l'autonomia a la independència. Barcelona, España: L'Avenç.

Rawls, J. (1996). El liberalismo político. Barcelona, España: Crítica.

Ruiz-Giménez, J. e Ruiz-Giménez, I. (2006). Comentario al Artículo 10 de la Constitución Española. Oscar Alzaga Villaamil, Santiago Sánchez González, et al. (dir.). Comentarios a la Constitución Española. Tomo II - Articulos 10 a 23 de la Constitución Española de 1978, Edersa. 
Vilajosana, J. M. (2014). Principi democràtic i justificació constitucional del dret de decidir, Revista d'Estudis Autonòmics i Federals, n. 19, pp. 178-210. Traducción castellana en el mismo número; versión inglesa: Josep M. Vilajosana (2014). "The democratic principle and constitutional justification of the right to decide", Catalan Social Sciences Review, 4, 2014, pp 57-80.

Vilajosana, J. M. (2014). La interpretación del derecho a decidir (5-IV-2014), El País.

Vilajosana, J. M. (2017). Identificación y justificación del Derecho (2ª ed.). Madrid, España: Marcial Pons.

Vilajosana, J. M. (2019). Laboratorio Cataluña. Legalidad, legitimidad y eficacia en el proceso político catalán, I. Lasagabaster (coord.): Crisis institucional y democracia (A propósito de Cataluña) (pp. 75-99). Valencia, España: Tirant lo Blanch.

Von Wright, G. H. (1971). Norma y acción. Una investigación lógica. Madrid, España: Tecnos. 